\title{
Variations
}

Variations Revue internationale de théorie critique

$24 \mid 2021$

Echos

\section{Lénine, Empiriocriticisme (1931)}

\section{Max Horkheimer}

\section{OpenEdition}

\section{Journals}

\section{Édition électronique}

URL : https://journals.openedition.org/variations/1974

DOI : 10.4000/variations. 1974

ISSN : 1968-3960

\section{Éditeur}

Les amis de Variations

\section{Référence électronique}

Max Horkheimer, «Lénine, Empiriocriticisme (1931) », Variations [En ligne], 24 | 2021, mis en ligne le 03 juillet 2021, consulté le 07 juillet 2021. URL : http://journals.openedition.org/variations/1974 ; DOI : https://doi.org/10.4000/variations.1974

Ce document a été généré automatiquement le 7 juillet 2021.

Les ami•e•s de Variations 


\title{
Lénine, Empiriocriticisme (1931)
}

\author{
Max Horkheimer
}

\section{NOTE DE L'ÉDITEUR}

Tapuscrit de Max Horkheimer, daté de 1931, rendu accessible au public en ligne par la section collections de la bibliothèque universitaire Johann Wolfgang Goethe, Francfort sur le Main, tous droits réservés : http://sammlungen.ub.uni-frankfurt.de/horkheimer/ content/pageview/6553861

Le livre de Lénine, Matérialisme et empiriocriticisme (1909) que Horkheimer discute ici venait à l'époque d'être publié en allemand dans la première édition des Euvres léniniennes, à Vienne. Toutes les notes de bas de pages sont établies par le traducteur, pour indiquer l'origine des références citées par Horkheimer.

\section{$[\ldots]$}

2 Le titre et le contenu du livre ${ }^{1}$ ne laissent pas planer le moindre doute sur son objet, la philosophie, en particulier les domaines très spécifiques de la philosophie qui concernent la philosophie de la connaissance. Le livre a été écrit pour contrer certaines opinions philosophiques précises qui ont pris place chez quelques dirigeants du parti (social-démocrate russe), à travers la philosophie de la connaissance de Mach et Avenarius. La propagande de ces dirigeants s'est caractérisée par l'idée qu'il fallait compléter les enseignements de Marx par l'empirio-criticisme ou les fonder sur cette approche. Ils professaient la nécessité de recourir à cette nouvelle philosophie, afin de permettre au marxisme de satisfaire certaines conceptions du monde, davantage qu'il n'a été capable de le faire jusqu'alors. Ce faisant, ils se sont moins placés dans une contradictions palpable avec quelques parties des textes rédigés par Marx qu'avec les versants philosophiques bien connues de l'oeuvre d'Engels. Tout le monde sait que Marx s'est plutôt consacré à l'étude de la théorie économique dans sa période tardive, tandis qu'Engels a examiné d'autres aspects du matérialisme historique au même moment, notamment dans l'Anti-Dühring et dans une série de petits articles, dont son étude au sujet du matérialisme historique. Il faut savoir que ces positions d'Engels ne 
doivent pas être considérées comme des opinions personnelles, mais qu'elles ont été acceptées dans leur principe par Marx, elles ont même parfois été co-rédigées par lui. Lénine a donc sans doute raison d'affirmer que là, où les défenseurs de l'empiriocriticisme entrent en friction avec les conceptions philosophiques d'Engels, ils entrent également en contradiction avec le marxisme en tant que tel. Avec une singulière colère, il signale à quel point il est significatif que ses adversaires se servent d'un effet de flou pour gommer cette contradiction. Ce n'est pas leur opposition à certaines thèses de Marx ou d'Engels qui serait en soi "a-marxiste", mais le gommage et l'occultation de principes de la théorie marxiste, ce qui les mènerait vers une interprétation lacunaire, insuffisante, qui mérite tout simplement d'être combattue. Lorsque, auparavant, des marxistes orthodoxes ont voulu corriger certains thèmes marxiens, la contradiction a toujours fini par apparaitre au grand jour. La dangerosité des empirio-critiques russes résiderait d'abord dans le fait qu'ils présentent les enseignements de Mach comme une sorte de signature marxiste et le marxisme sous la lumière de la théorie de Mach, pour faire croire que tout cela n'est qu'une seule et même chose. En conséquence, l'intention de Lénine est double: d'abord la mise en relief de la contradiction entre empiriocriticisme et théorie marxiste; puis la défense de la seconde, où elle permet de contrer l'empiriocriticisme, afin de le diminuer par la contre-attaque.

3 Je vais dans un premier temps restituer la manière dont ces deux dimensions sont traités, ce qui permet de résumer l'essentiel du livre. Ensuite, je vais m'efforcer de formuler une critique des argumentations léniniennes, afin de définir, finalement, l'enjeu strictement programmatique qui reste encore à résoudre, après s'être manifesté dans l'objet que traite Lénine.

4 Toute la démonstration de Lénine, concernant la première dimension, se fonde sur l'opposition idéalisme/matérialisme. La référence d'Engels que Lénine mobilise de manière récurrente : Feuerbach, p. 13-15.

Chez Lénine, la contradiction est formulée de manière moins universelle que dans l'original: il voit le matérialisme comme une conception, où "la nature, la matière, constituent l'origine", alors que l'idéalisme y apparait comme la conception qui voit "l'esprit, la conscience, la perception" à l'origine de tout. Ou encore, pour l'exprimer avec les mots qui caractérisent le discours de Lénine (p. 39), la lignée philosophique de l'idéalisme irait des perceptions sensibles "vers le monde extérieur", alors que la lignée matérialiste irait du monde extérieur vers les perceptions sensibles. Ainsi, le matérialiste considère la matière comme la chose première, et la conscience comme étant secondaire, et ce serait tout le contraire pour l'idéaliste.

6 Dans quelle mesure Mach doit-il donc être considéré comme un idéaliste ? - L'exposé de la théorie de Mach doit tenir compte de l'introduction de Lénine, selon laquelle cette théorie ne serait qu'une variété actualisée du berkeleyanisme. Partant de la doctrine de Berkeley, qui voudrait que les choses ne sont que des compositions de la perception subjective.

7 Basé sur l'exposé de Berkeley, apparaissent successivement les duplications, les écarts et innovations dans la théorie de Mach ou Avenarius (théorie élémentaire), des écarts que Lénine juge peu significatifs.

[...] 
Il s'agit d'un idéalisme, au sens où les expériences conscientes sont la seule source de la connaissance et sont son seul objet. Il y a distinction avec le Berkeley théologique, et affinité avec Hume.

Maintenant, passons à la question de savoir qui a raison? Pour conclure la réflexion précédente: Mach et Avenarius sont des idéaliste, mais d'un nouveau genre. Lénine n'a pas aperçu l'opposition décisive entre Mach à Berkeley, dans les deux variétés d'idéalisme qui sont en jeu. Dans le langage employé par Lénine, un gros mot devrait ici servir de commentaire. Il aurait du être alerté par la fonction historique complètement différente des deux théories: le combat de Berkeley contre l'athéisme aux côtés de l'église intégrée à la société de cour, assorti de la réaction consciente, la téléologie; il veut précisément sauver "les esprits" et les âmes. En revanche, Mach incarne l'espoir des petits bourgeois d'arriver à un progrès terrestre, d'où leur idéal de progrès, dont nous ne pouvons pas examiner la signification à cet endroit. Mais il est parlant que Lénine examine les théories dont il s'agit, en faisant complètement abstraction de ces aspects. Nous y reviendrons. Il n'a pas compris la différence historique et théorique.

Venons-en à la deuxième dimension : le fond de la querelle.

Partons de l'énumération des reproches que Lénine adresse à la théorie de Mach, ou au Machisme comme il le dit aussi.

1. (déjà évoqué plus haut) Mach ne fait que plagier Berkeley² ${ }^{2}$ et même Fichte!

Citation de Lénine : Les différentes façons de s'exprimer de Berkeley en 1710, de Fichte en 1801 et d'Avenarius en 1891-1894 ne changent rien au fond, c'est-à-dire à la tendance philosophique essentielle de l'idéalisme subjectif. ${ }^{3}$ Mach semble aussi plagier Hume et d'une manière générale tous les idéalistes subjectivistes. C'est une drôle de manière de s'exprimer, que de stigmatiser comme des coupables de plagiat tous les auteurs d'un certain courant de pensée qui se réfère à ses prédécesseurs.

2. Toutes les formes d'idéalisme subjectif, celui de Mach inclus, doivent nécessairement s'enferrer dans un solipsisme ${ }^{4}$ : Berkeley a suffisamment démontré en son temps qu'«avec des sensations, c'est-à-dire avec des éléments psychiques», on ne peut "construire» rien d'autre que le solipsisme. ${ }^{5}$ - Il s'agit ici d'une confusion avec l'illusionnisme : La doctrine selon laquelle les corps sont des complexes de sensations, etc. est un illusionnisme absolu, c'est-à-dire un solipsisme, puisque l'univers n'est, de ce point de vue, que mon illusion. ${ }^{6}$

3. Mach entrerait en collision avec les sciences naturelles, qui doivent nécessairement partir de présupposés matérialistes (ex. la géologie; plus généralement apparait la conception que toutes les expériences sensibles ne sont que des images représentatives de la réalité).

4. Mach ferait entrer, en contrebande, l'affirmation matérialiste dans son système, selon laquelle les perceptions sensibles sont liées à l'existence d'une matière organique.

5. Mach gomme la contradiction idéalisme/matérialisme, par un exploit artistique qui passe par le mot "élément" (déjà évoqué).

6. Mach n'arrive pas à expliquer que la terre a existé avant l'humanité (lire p. 62).

Tout idéalisme subjectif est un moteur du fidéisme, car si les choses ne sont que des ensembles de perception, alors nous ne pouvons en aucune façon contester l'objet de la croyance de ceux qui sont croyants. Malheureusement, Lénine ne s'exprime ici pas d'une manière aussi claire qu'il le pourrait; en tout cas il a raison de dire qu'il n'y a qu'un petit pas qui sépare l'idéalisme du fidéisme. 
21 Mais - et c'est là la tâche la plus importante à laquelle nous sommes confrontés - la question reste de savoir dans quelle mesure l'affirmation de Lénine échappe à cette même accusation. Et cela, alors qu'il dresse la philosophie matérialiste en face de l'idéalisme dans son livre, de manière positive.

Nous le savons, puisque Lénine lui-même insiste fréquemment sur ce point, que les matérialistes se voient confrontés à l'accusation de verser dans la métaphysique et dans une croyance naïve, cette même accusation qu'ils avaient adressé aux adversaires idéalistes.

Lire les passages p. 113-115, puis p. 117-118 pour voir ce point de vue de Lénine.

La théorie des images représentatives. ${ }^{7}$ Elle se trouve chez Marx et Engels - puis chez Lénine. Chez les deux premiers, elle est d'abord complexe, et plus tard élaborée de manière précise. Lénine voit (p.117) une réalité objective donnée à l'homme dans ses sensations qui la copient, la photographient, la reflètent, et qui existe indépendamment des sensations. ${ }^{8}$ Nous trouvons une réponse très précise chez Lénine, à la question de savoir ce qui est l'objet des représentations qui se reflètent ainsi : la matière, l'espace et le temps. Le contre-argument - celui qui relève que nous ne pouvons pas définir exactement ce qu'est la matière, ainsi que l'espace et le temps, puisque les théories que produit l'humanité pour saisir cela ne cessent d'évoluer - a été réfuté par Lénine de manière efficace à travers sa conception de la vérité relative et absolue. Lire, après une clarification des concepts la page 167. Et qui bute aussitôt sur des complications, p. 169.

Critique de la théorie des images représentatives, et passage à une critique globale de la théorie de Lénine.

26 Le point cardinal de la critique : la relation du sujet et de l'objet, de la conscience et de la matière, est traitée de manière complètement non-dialectique, comme s'il s'agissait de poser un rapport fixe qui resterait valable pour l'éternité. La relation du sujet et de l'objet n'est pas saisie en tant que praxis humaine, malgré le fait que cette praxis ne cesse d'être invoquée, comme nous allons le montrer. Ici se trouvent postulés des principes doctrinaires absolus, qui échappent à tout critère de jugement. Des expérimentations peuvent servir à élaborer des critères, à partir desquels est jugée une réalité circonscrite, et cette réalité seulement, mais elles ne peuvent pas fonder des postulats métaphysiques qui servent de cadre aux argumentations de Lénine. C'est pourquoi il devient la proie de ses adversaires - à partir du point décisif qu'il ne parvient pas à reconnaitre, ni à intégrer dans sa critique - il s'est laissé piéger par eux en se laissant attirer sur leur propre terrain. Face à leurs postulats, il affirme lui-même ses propres principes dogmatiques : il existerait une matière de manière absolue, un espace et un temps absolu, rien n'existe en dehors de l'espace et du temps; la conscience représenterait la réalité en la suivant de près, tel un appareil photographique, ou d'autres appareillages qui furent déjà l'objet des moqueries de Marx et Engels à l'égard d'un certain matérialisme. Lénine converge ainsi avec tous les "réalistes" de facture métaphysique, en particulier avec les réalistes réactionnaires contemporains.

27 C'est l'origine d'une grave confusion, qui ne frappe pas d'abord Lénine, mais ceux qui ont voulu éditer cet écrit comme étant le premier volume de ses œuvres. En 1908, Lénine avait l'intention de combattre une mauvaise philosophie au sein du parti (socialdémocrate) russe, qui lui paraissait dangereuse au vu de l'évolution du marxisme en Russie. Dans ce but, il a écrit un gros livre, qui donne à voir sa manière caractéristique 
de diriger passionnément toute son énergie vers une seule tâche, après avoir survolé toute la littérature principale. Je ne pense pas me tromper entièrement si je rappelle le constat, qu'à cette époque valait l'adage que le poids du contenu d'un livre devait être en relation avec son nombre de pages. Au fait, je connais de bien meilleurs morceaux philosophiques de Lénine, je me souviens en particulier d'un article de janvier 1921 à l'encontre de Trotsky et Boukharine au sujet de la question syndicale, publié dans le second numéro de la revue Unter dem Banner des Marxismus', et à son fragment sur la question de la dialectique..$^{10}$ En tout cas, Lénine a atteint son but, à travers son livre Matérialisme et empiriocriticisme, car les supporteurs de Mach ont perdu beaucoup de leur influence par la suite. Mais aujourd'hui, l'on nous présente ce livre comme un texte d'actualité, assorti d'une introduction de Déborine ${ }^{11}$ qui proclame que de nos jours, en Allemagne et en Autriche, la scolastique kantienne et l'idéalisme positivste seraient... en pleine floraison. Loin de là! Déborine donne l'impression d'habiter sur la face opposée de la Lune, il est misérablement mal renseigné sur les conditions réelles. En Allemagne, l'idéalisme positiviste est devenu une sorte d'insulte - sans le concours du livre de Lénine - et un docteur en philosophie qui est stigmatisé comme étant un idéaliste positiviste aura la plus grande peine d'obtenir son habilitation dans la plus petite université, je doute même du fait que l'on puisse encore soutenir pareille thèse de doctorat. Non - en ce sens-là, le livre n'est en rien actuel, il ne fait qu'enfoncer des portes ouvertes. Mais c'est bien là le problème, il s'agit de voir que le livre ne se distingue de la philosophie académique aborde que par sa piètre qualité, par sa méthode moins élaborée, sa facture plus primitive. Marx n'avait non seulement une longueur d'avance sur la philosophie la plus raffinée de son époque (Hegel!), tout en maniant son appareillage conceptuel comme s'il s'agissait de son propre instrument, mais il était même en avance en rapport à la totalité des théories disponibles de son temps. Lénine se laisse entrainer sur le terrain de ses adversaires, il pose un dogme contre un autre dogme, une croyance contre une autre, ce qui fait qu'il reste bien en arrière de sa propre faculté, de sa propre théorie, et encore plus en rapport à ses professeurs Feuerbach, Marx et Engels.

Je vais essayer d'étayer cette idée. Dès le début, j'ai annoncé que j'allais conclure en montrant dans quelle direction il faudrait s'engager pour avancer dans la lutte contre les doctrines idéalistes, par exemple cette de Mach. Je précise que l'orientation que je propose prend à son compte la méthode marxiste. Lénine, lui, affirme ses propres convictions philosophiques, en face de son adversaire. Un peu comme un philosophe d'aujourd'hui, qui postule sans transition qu'il existe un dieu personnel et une vérité absolue, pour montrer aussitôt qu'il n'existe aucun dieu personnel et aucune vérité absolue. Ainsi, Lénine prétend qu'il existe une matière absolue, et une vérité absolue qui serait composée de la somme des vérités relatives. De son point de vue, le Machisme qui juge que le tout serait composé des éléments du monde, se présente comme une pitoyable doctrine professorale, comme "une soupe éclectique pour mendiants". Je ne suis pas le seul à penser que cela ne clôt pas le débat, il n'est pas possible d'avancer de cette manière, même si de telles proclamations irriguent tout un livre volumineux, où les attaques sont déclinées à l'encontre d'un ensemble de disciples de Mach aux noms plus ou moins connus, d'obédience russe, française, anglaise ou allemande.

Un marxiste a écrit : "La logique dialectique exige d'aller plus loin. Afin de saisir un objet, il convient d'étendre la recherche sur toutes ses dimensions, toutes ses relations et toutes ses médiations. Nous n'allons y parvenir complètement, mais l'exigence d'une analyse complète nous met en garde contre des erreurs et contre l'ossification. (...). Troisièmement, la 'définition' 
exhaustive d'un objet doit prendre en charge toute la praxis humaine, en tant qu'un critère de la vérité, et en tant que caractérisation de la relation entre l'objet et des besoins humains. Quatrièmement, la logique dialectique nous apprend qu'une 'vérité abstraite n'existe pas, que la vérité est toujours concrète', comme le regretté Plechanov le disait à la suite de Hegel. ${ }^{12} \mathrm{Le}$ marxiste que je viens de citer n'est personne d'autre que Lénine lui-même, qui écrit cela à la suite de son discours à l'encontre de Trotsky et Boukharine que j'ai déjà évoqué. Lénine n'a rien retenu de cette méthode dialectique, concernant la doctrine de Mach. Il n'a pas avancé dans la recherche des relations et médiations qui font partie de la réalité sociale, et il n'a donc pas su éviter "erreurs" et "ossification". Il ne restitue pas non plus le mouvement ou le développement en soi de la philosophie allemande - ou disons plus précisément que l'éditeur Déborine n'a pas la moindre idée de ce développement. Il n'est plus question de prendre en charge toute la praxis humaine, afin de saisir l'objet, car Lénine traite l'empiriocriticisme et tout l'idéalisme subjectif de manière complètement isolée, en tant que simple théorie philosophique, sans jamais faire l'effort de le comprendre à partir de la pratique humaine. Il aborde le tout en tant que "vérité abstraite" et y oppose sa "vérité abstraite", alors que la vérité est "toujours concrète" selon les mots de Plekhanov, Hegel et de Lénine lui-même.

A cet endroit, je signale ce qui aurait dû être accompli du point de vue d'une critique marxiste. Toutes les théories, idées métaphysiques, doctrines et conceptions du monde, sont, comme le dit Marx, directement et intimement mêlée à l'activité matérielle et aux échanges matériels des hommes, elle est le langage de la vie réelle. (...) Ce sont les hommes qui sont les producteurs de leurs représentations, de leurs idées, etc., mais les hommes réels, agissants, tels qu'ils sont conditionnés par un développement déterminé de leurs forces productives et des rapports qui y correspondent, y compris les formes les plus larges que ceux-ci peuvent prendre. (...)

31 Et même les fantasmagories dans le cerveau humain sont des sublimations résultant nécessairement du processus de leur vie matérielle que l'on peut constater empiriquement et qui repose sur des bases matérielles (...) Ce n'est pas la conscience qui détermine la vie, mais la vie qui détermine la conscience. En ce sens, il s'agit de partir d'une conception "qui correspond à la vie réelle, on part des individus réels et vivants eux-mêmes et l'on considère la conscience uniquement comme L e u r conscience. ${ }^{13}$

Il eut fallu montrer, comment, au milieu de la réalité sociale de l'Allemagne du début de notre siècle, la philosophie petite bourgeoise de Mach a pu émerger, puis sous quelles conditions elle a pu trouver une résonance dans certains milieux russes, et saisir la manifestation d'une situation sociale qui s'exprime dans une telle situation, etc. Lénine aurait alors pu trouver - je me contente d'en esquisser ici la possibilité en l'absence de telles études - ou il aurait éventuellement pu dire qu'une telle philosophie de la connaissance peut correspondre à une petite-bourgeoise qui tente de s'autonomiser, qui place le critère de la connaissance à l'intérieur du domaine de perception de chaque sujet individuel, et qui identifie le monde par la conscience bourgeoise, ne croyant que ce qui est palpable. Pareille petite-bourgeoisie veut alors croire en ses chances d'ascension sociale au sein de la société, et elle se distingue par une croyance du progrès pour les membres individuels de sa classe, cela d'une manière bien plus nette qu'elle n'a pu l'exprimer par la suite, lorsqu'elle va être confrontée à la domination des trusts et monopoles. Lénine aurait alors pu indiquer, peut-être, que la philosophie de Mach était déjà un peu en retard sur son temps, qu'elle en était une forme posthume, et 
qu'il y avait de bonnes raisons qu'elle soit disputée plus âprement en Russie qu'en Allemagne.

J'insiste sur la fait qu'une telle étude n’a pas encore été réalisée.

\section{NOTES}

1. Ndt: Il s'agit probablement de la première édition allemande des œuvres de Lénine publiée à Vienne, dont: Lenin, Materialismus und empiriocritizismus, Wien Verlag für Literatur und Politik 1925.

2. Ndt: W.I.Lénine, Matérialisme et empiriocriticisme, ES, 1975, p.16 : Nous avons reproduit intégralement le paragraphe 6 de «remarques antimétaphysiques» de Mach. Ce n'est d'un bout à l'autre qu'un plagiat de Berkeley.

3. Ndt: op.cit.

4. Ndt: définition du solipsisme (Larousse): Conception selon laquelle le moi, avec ses sensations et ses sentiments, constitue la seule réalité existante dont on soit sûr.

5. Ndt: Lénine, op.cit.

6. op.cit., p.27.

7. Ndt: le terme image représentative (Abbild) ou image mentale (Gedanken-Abbild) se trouve dans les textes d'Engels et les éditions allemandes d'autres auteurs auxquels se réfèrent Engels, Lénine ou Horkheimer à la base, et donc également dans l'édition allemande du texte de Lénine (Materialismus und Empiriokritizismus, 1908/1925). Nous parlons d'images représentatives (pour Abbild) et non d'image (Bild) pour rendre la distinction conceptuelle qui existe en allemand, entre les mots Bild et Abbild. Lénine était germanophone. Nous gardons la traduction française d'image mentale pour Abbild.

8. Lénine, op.cit. Citation de la phrase complète de Lénine utilisé par Horkheimer dans sa version allemande: Die Materie ist eine philosophische Kategorie zur Bezeichnung der objektiven Realität, die dem Menschen in seinen Empfindungen gegeben ist, die von unseren Empfindungen kopiert, fotografiert, abgebildet wird und unabhängig von ihnen existiert.

9. Ndt: Sous la bannière du marxisme fut une revue intellectuelle émanant de la jeune Internationale communiste, publiée dans plusieurs langues. L'article de Lénine, en réponse à un texte de Trotsky sur le rôle des syndicats, porte sur La situation présente et les erreurs de Trotsky et Boukharine, mais pour trouver la partie philosophique qui intéresse Horkheimer, il faut se référer à une version plus complète qui se trouve dans la version allemande de oeuvres de Lénine: Lenin, Werke, Dietz, vol. 32.

10. Ndt: Le fragment sur la dialectique figure dans Lénine, Résume de la science de la logique de Hegel, 1914, oeuvres vol. 38 (disponible en ligne).

11. Ndt: Abram Déborine était un théoricien central du marxisme soviétique depuis la prise de pouvoir de Staline, mais qui l'écarta finalement en 1931 par lui. Il existe une préface de Déborine au livre de Lénine aux éditions Wien Verlag für Literatur und Politik (l'édition viennoise peut expliquer l'allusion de Horkheimer à l'Autriche).

12. Lenin, Werke, Dietz, vol. 32, pp.84-85.

13. Ndt: Horkheimer omet de mentionner la source de ces citations, qui est évidente: K.Marx, FEUERBACH (1848) in : Marx/Engels, MEGA, vol. 5, De Gruyter, 2016 (citations tirées de la 
traduction française portant le titre - qui est celui de l'édition soviétique de 1931- L'idéologie allemande, ES, 1952. 